\title{
Persistent threats to validity in single-group interrupted time series analysis with a cross over design
}

\section{Ariel Linden $\mathrm{DrPH}^{1,2}$}

\author{
${ }^{1}$ President, Linden Consulting Group, LLC, \\ Ann Arbor, Michigan, USA \\ ${ }^{2}$ Research Scientist, Division of General \\ Medicine, Medical School, University of \\ Michigan, Ann Arbor, Michigan, USA \\ Correspondence \\ Ariel Linden, Linden Consulting Group, LLC, \\ 1301 North Bay Drive, Ann Arbor, MI 48103, \\ USA. \\ Email: alinden@lindenconsulting.org
}

\begin{abstract}
Rationale, aims and objectives The basic single-group interrupted time series analysis (ITSA) design has been shown to be susceptible to the most common threat to validity-history -the possibility that some other event caused the observed effect in the time series. A singlegroup ITSA with a crossover design (in which the intervention is introduced and withdrawn 1 or more times) should be more robust. In this paper, we describe and empirically assess the susceptibility of this design to bias from history.

Method Time series data from 2 natural experiments (the effect of multiple repeals and reinstatements of Louisiana's motorcycle helmet law on motorcycle fatalities and the association between the implementation and withdrawal of Gorbachev's antialcohol campaign with Russia's mortality crisis) are used to illustrate that history remains a threat to ITSA validity, even in a crossover design.
\end{abstract}

Results Both empirical examples reveal that the single-group ITSA with a crossover design may be biased because of history. In the case of motorcycle fatalities, helmet laws appeared effective in reducing mortality (while repealing the law increased mortality), but when a control group was added, it was shown that this trend was similar in both groups. In the case of Gorbachev's antialcohol campaign, only when contrasting the results against those of a control group was the withdrawal of the campaign found to be the more likely culprit in explaining the Russian mortality crisis than the collapse of the Soviet Union.

Conclusions Even with a robust crossover design, single-group ITSA models remain susceptible to bias from history. Therefore, a comparable control group design should be included, whenever possible.

\section{KEYWORDS}

causal inference, crossover design, interrupted time series analysis, quasi-experimental

\section{1 | INTRODUCTION}

Single-group interrupted time series analysis (ITSA) is a popular evaluation strategy for observational data in which a single unit is studied (eg, an individual, a city, or a country), the dependent variable is a serially ordered time series, and multiple observations are captured in both the pre- and postintervention periods. The study design is called an interrupted time series because the intervention is expected to "interrupt" the level and/or trend of the time series, subsequent to

[Correction added on 17 November 2016, after first online publication: The article's title has been edited due to similarity with another of the author's published article.] its introduction. ${ }^{1,2}$ It has been maintained that ITSA generally has strong internal validity, primarily through its control over regression to the mean ${ }^{1-4}$ and good external validity, particularly when the analysis occurs at the population level, or when the results can be generalized to other units, treatments or settings. ${ }^{2,5}$

Recently, the validity of the basic single-group ITSA design (consisting of a preintervention phase and an intervention phase) has been scrutinized. Linden ${ }^{6}$ illustrated that this design can either fail to identify the effects of external factors on the time series, resulting in a false causal attribution, or conversely confuse the causal interpretation when a directionally correct change in the time series also occurs before the intervention. In both cases, the inclusion of a comparable control group clarifies causal effects. 
Shaddish et $\mathrm{al}^{2}$ suggest adding design features such as the removal of the treatment at a known time, or by extension, adding multiple replications, to improve the validity of the single-group ITSA design. In essence, this is a single-group version of the crossover design, in which the intervention is introduced and withdrawn, 1 or more times. The underlying premise is that it would be increasingly unlikely that external events will affect the time series coincidentally with each successive crossover, and thus, the results can be considered a causal effect of the intervention if the time series changes accordingly. ${ }^{2}$

The purpose of the current paper is to offer a nontechnical discussion of how, even with the addition of a crossover design, factors other than the intervention may be mistaken for a treatment effect (or withdrawal of treatment) when only a single group is being evaluated. By way of example, it will be shown that the effects of external events can only be identified and controlled for by utilizing a comparable control group to serve as the counterfactual-a fundamental element of the potential outcomes framework. ${ }^{7,8}$ With the inclusion of a comparable control group, factors other than the intervention that are responsible for shifting the time series in each crossover phase will likely be observed in both groups and thus, not mistaken for an effect of treatment or withdrawal. Likewise, directionally correct changes that do occur in the intervention group but not in the control group may be interpreted as causal. ${ }^{9,10}$ This problem is illustrated using data from 2 natural experiments: the effect of multiple repeals and reinstatements of Louisiana's motorcycle helmet law on motorcycle fatalities and the association between the implementation and the withdrawal of Gorbachev's antialcohol campaign and Russia's mortality crisis in the early 1990s.

\section{2 | THREATS TO VALIDITY IN THE BASIC AND CROSSOVER SINGLE-GROUP ITSA DESIGNS}

Although the basic single-group ITSA design (consisting of a preintervention and intervention phase) can control for many threats to validity, the remaining threats that the design does not control for are critical. $^{1,2}$ Consequently, investigators typically add features to the single-group design, such as 1 or more treatment crossovers, with the intent of mitigating the influence of these remaining biases.

History is the possibility that some event other than the intervention caused the observed effect in the time series, ${ }^{2}$ and it is the principal threat to validity of any single-group ITSA design. There are at least 2 scenarios where the effect of history may be overlooked or misinterpreted. First, some factor may cause a directionally correct change in the time series before the intervention. As such, any additional change in the time series subsequent to the introduction of the intervention may be considered a continued or magnified effect of that previous factor rather than a treatment effect. ${ }^{9}$ Recently, sensitivity tests adapted from the regression-discontinuity literature ${ }^{11}$ have been applied to the ITSA design to identify these false treatment effects. ${ }^{12}$ In the second scenario, the change in the time series after initiation of the intervention is immediate and drastic, and as such, it is easy to ignore the possibility that some other factor may be the cause. Even if there is an alternative explanation for the effect, information may not always be available to identify those factors. Thus, the investigator is likely to argue that the effect is causally related to the intervention without further study. ${ }^{6}$

A crossover design is considered a more robust approach to control for history than the basic single-group ITSA, by virtue of the fact that there are more comparisons involved, making it less likely that some external event will affect the time series repeatedly at each crossover phase. For example, adding a specific time point when the treatment is deliberately withdrawn allows the investigator to evaluate change in the time series between 3 distinct phases (preintervention vs intervention phase, intervention vs postintervention, and preintervention vs postintervention). Conversely, in a simple ITSA design, the intervention is considered ongoing, thereby limiting the comparison to only the preintervention and intervention phase. If the time series changes in the expected direction after each crossover, then it may be harder to argue that the effect was caused by some external event. In theory, as more crossovers are added to the study, the threat of history should diminish accordingly. However, other external events may in fact be causing changes in the time series coincidentally with the initiation or withdrawal of the intervention, regardless of the number of crossovers. Only with the inclusion of a control group for comparison, will the effect of external events on the time series be identified.

Although history is the most common threat to validity, there are at least 3 other threats to which ITSA is susceptible. Instrumentation, or a change in how the time series is measured, is a threat to validity that may erroneously appear as a treatment effect in both the basic single-group ITSA and crossover design. ${ }^{2}$ As an example, in health management interventions, patients' health behaviors are sometimes measured on different scales over time or a particular scale may be altered. ${ }^{13,14}$ As a result, the measurements will be both inconsistent and unreliable. In general, although documentation should be obtained indicating how and when the instrumentation changed, it may nevertheless be impossible to control for this bias in either a single-group ITSA or crossover design. However, with the inclusion of a comparable control group, the change in instrumentation should affect both time series equally, thereby nullifying its effect.

Selection may bias both the single-group ITSA and crossover design if the serial observations are cross-sectional and the characteristics (or composition) of the group under study are different in any 2 (or more) crossover phases of the study (selection is not a factor in either a single-group ITSA or crossover design where the same group, or individual, undergoes surveillance over the duration of the study). Selection may be controlled for by finding a control group that is comparable with the treatment group on preintervention characteristics (at the very least, the groups should be comparable on the preintervention level and trend of the outcome under study). ${ }^{9,10}$

Threats to statistical conclusion validity common to any study design also apply to ITSA. These include low power, violated test assumptions, and unreliability of measurement. ${ }^{2}$ Although these issues are important, their discussion is beyond the scope of this paper. The reader is referred to Box and Tiao, ${ }^{15}$ Glass et al, ${ }^{16} \mathrm{McD}$ owall et al, ${ }^{17}$ Crosbie, $^{18}$ Gottman, ${ }^{19}$ Linden, ${ }^{9}$ Linden and Adams, ${ }^{10}$ McKnight et al, ${ }^{20}$ Simonton, ${ }^{21}$ and Velicer and McDonald. ${ }^{22}$

In the following 2 empirical examples, we demonstrate the susceptibility of the single-group ITSA crossover design to bias from history. 
In the first example, the data are analyzed as a single-group ITSA where the estimated effects substantiate the hypothesized effects. Next, the time series for the intervention group is contrasted with that of a control group to see if the treatment effects still stand. If the time series for both groups are similar across all phases, then we have demonstrated that the single-group ITSA results are biased because of history (because events outside of the intervention are affecting the control group as well). In the second example, the data are analyzed as a single-group ITSA where the estimated effects substantiate the hypothesized effects. However, given that our focus in this example is on the cause of the spike in the time series at the time of the intervention's withdrawal, the time series for the intervention group is contrasted with that of several different control groups as sensitivity analyses. History may be the source of bias if the time series spikes in control groups where we hypothesize they should remain stable (because an external event was causing a spike in both groups similarly).

\section{3 | EXAMPLE 1: THE EFFECT OF MULTIPLE REPEALS AND REINSTATEMENTS OF LOUISIANA'S MOTORCYCLE HELMET LAW ON MOTORCYCLE DEATHS}

Louisiana first enacted a universal motorcycle helmet law in 1968. Then in 1976, the law was partially repealed to require helmet use only by riders younger than 18 years. In 1982, the universal helmet law was reinstated. In 1999, the law was amended to require helmet use only by motorcyclists younger than 18 years and riders older than 18 years who did not have a minimum of $\$ 10,000$ in medical insurance coverage. In 2004, the universal helmet law was again reinstated. ${ }^{23}$ This unusual policy situation of multiple repeals/reinstatements (4 distinct phases) of the law provides an excellent opportunity for testing the validity of the single-group ITSA crossover design to assess the effect of the helmet law (and its repeal) on motorcycle fatalities.

For the current analysis, all motor vehicle fatality data for all states were retrieved from the Fatal Accident Reporting System database for the years 1975 to 2014 (which is all the data available in the system). ${ }^{24}$ Annual issues of Highway Statistics provided motorcycle registration data for the periods of 1996 to 2001, and years between 1975 and 1996 were retrieved from the 1995 summary volume. ${ }^{25}$ Statistical analyses were conducted using ITSA, a program written for Stata 14.1 (StataCorp., College Station, TX, USA) to conduct single-group and multiple-group interrupted time series analyses. ${ }^{9,10}$ When comparisons are made between times series on different scales or at different levels of the same scale, the time series were ipsatively standardized ${ }^{26}$ to allow for comparisons on the same scale.

Figure $1 \mathrm{~A}$ presents the raw motorcycle annual fatality counts in Louisiana from 1975 to 2014. As shown, motorcycle deaths increased from 1975 to 1982, the period in which the helmet law applied only to riders younger than 18 years. Motorcycle deaths decreased annually from 1982 to 1999, the period in which the universal helmet law was reinstated. Motorcycle deaths increased annually from 1999 to 2004 , the period in which the helmet law was once again applied only to riders younger than 18 years, and annual motorcycle deaths
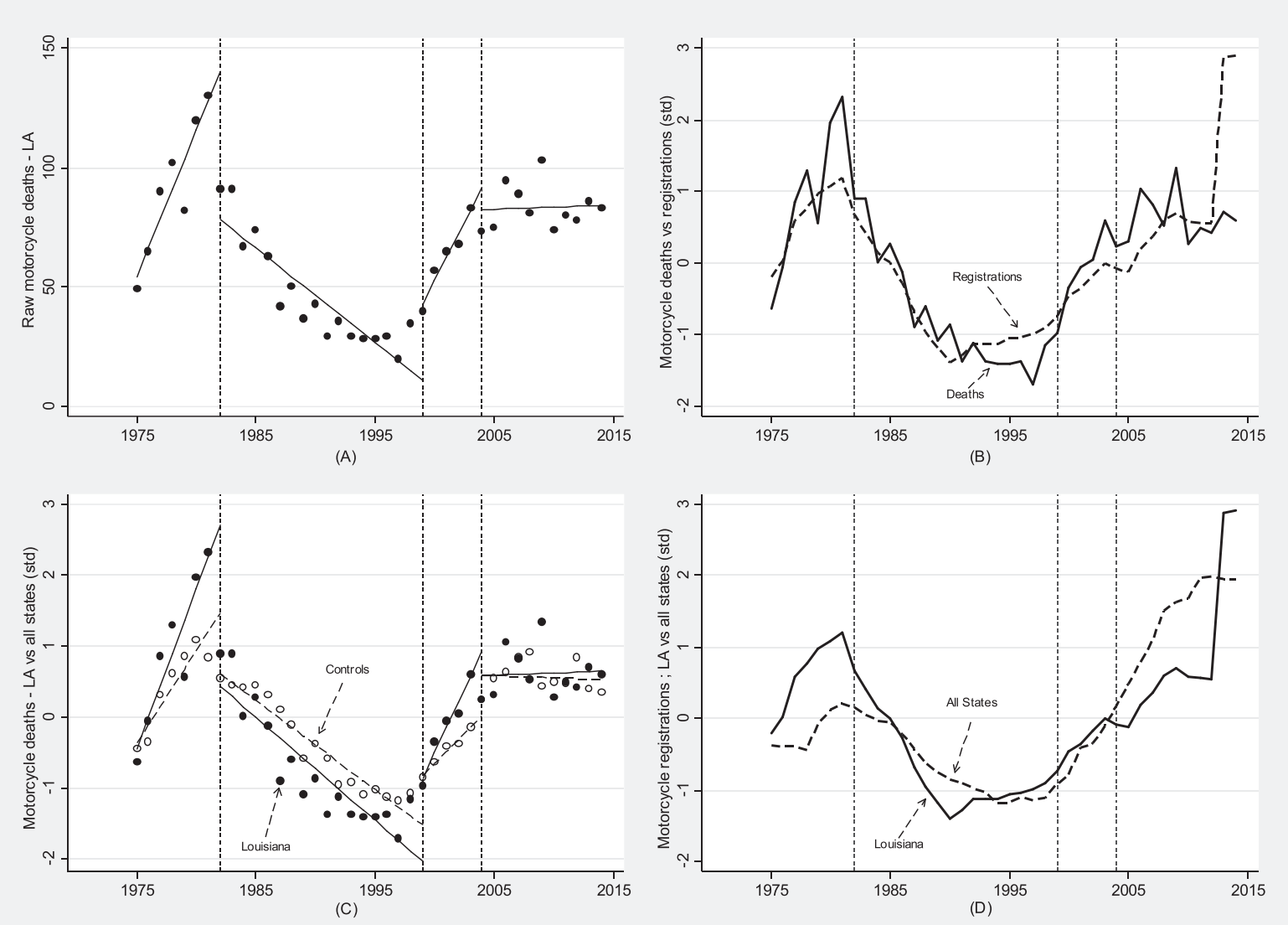

FIGURE 1 Louisiana motorcycle deaths and registrations from 1975 to 2014, using single-group and multiple-group ITSA designs 
appeared to stabilize (ie, the linear trend was flat) between 2004 and 2014, the period in which the universal helmet law was once again reinstated.

As clearly illustrated in Figure 1A, the annual motorcycle fatalities were directionally consistent with the expected effect. That is, Louisiana's multiple helmet law repeals were associated with increased motorcycle fatalities, and the universal helmet law reinstatements were associated with reduced or flat annual fatalities. In addition, one could argue that most likely threats to validity ${ }^{2}$ could be ruled out. For example, there were a sufficient number of annual observations in each treatment phase (ie, repeal and reinstatement periods) to rule out regression to the mean as a rival explanation. ${ }^{3,4}$ Selection bias may pose a threat to validity if the characteristics of those who died in each treatment phase differed systematically from those who died in the alternate treatment phase, with the most likely case being made for differential fatality rates based on the age cutoff of 18 years. However, an analysis by age-group did not bear that out (data not shown). History is a plausible threat to validity only if another event or action had occurred concomitantly with the initiation of each treatment phase. However, it is easy to dismiss the possibility that any other factor could have caused the effect outside of the policy changes, given such an immediate and dramatic effect that occurs simultaneously with the initiation of each and every treatment phase.

However, Figure 1B casts doubt on the hypothesis that the repeated repeals of the helmet law in Louisiana caused the increase in motorcycle deaths. As shown, motorcycle registrations followed a nearly identical historic pattern as motorcycle deaths, with the exception of the last few years of the data set, where a change in methodology for estimating registered vehicles may have distorted the reporting of registrations (a good example of instrumentation ${ }^{1,2}$ ). In light of these data, one may revise the previous hypothesis to now consider that motorcycle registrations hold the primary association with motorcycle deaths in Louisiana, rather than the repeal and reinstatement of the helmet law.

Figure $1 \mathrm{C}$ offers a complete rebuttal for any causal association between Louisiana's multiple helmet law repeals and reinstatements and the change in motorcycle fatalities. Here, standardized motorcycle fatalities in Louisiana are compared with those of all other States (excluding Arkansas, Florida, Kentucky, Michigan, Pennsylvania, and Texas-states that repealed their helmet laws during some point in the same timeframe under study). As shown, the general behavior of the time series in Louisiana is similar to that of all other states, irrespective of the multiple repeals and reinstatements of the universal helmet law in Louisiana.

Figure 1D illustrates standardized motorcycle registrations in Louisiana and the control states over the entire observation period. When paired with the corresponding motorcycle fatalities presented in Figure $1 \mathrm{C}$, the close relationship between registrations and fatalities remains evident (until the mid-2000s, when the change in methodology for estimating registrations began to distort the measurement of motorcycle registrations (for the details of the methodological changes, see http://www-fars.nhtsa.dot.gov/common/ FARS\%20Encyclopedia\%20VMT\%20Changes.pdf).

In summary, despite improving the robustness of the single-group ITSA design by including multiple treatment crossovers (ie, repeal and reinstatement), the effects of history still biased the results of the evaluation. Moreover, this bias was only revealed when Louisiana's time series was contrasted with that of the control states. The results of this analysis suggest that motorcycle fatalities are not causally related to the helmet law (ie, they do not decrease as a result of the law being enacted/reinstated, and they do not increase as a result of the law being repealed). However, given that motorcycle fatalities are so closely associated to motorcycle registrations, an alternate hypothesis may simply be that with more motorcycles on the road, there will be a likewise increase in the number of fatalities.

\section{4 | EXAMPLE 2: THE ASSOCIATION BETWEEN GORBACHEV'S ANTIALCOHOL CAMPAIGN AND RUSSIA'S MORTALITY CRISIS}

In 1985, Mikhail Gorbachev initiated an antialcohol campaign throughout the Soviet Union. The campaign was unprecedented in scale and scope, and it operated through both supply-side and demand-side channels, simultaneously raising the effective price of drinking and subsidizing substitutes for alcohol consumption. At the height of the campaign, official alcohol sales had fallen by as much as two thirds. In practice, the campaign lasted beyond its official end in 1988, as restarting state alcohol production took time, and alcohol prices remained elevated. ${ }^{27}$

All-cause mortality in the Soviet Union decreased during the campaign years but rose precipitously between 1990 and 1994 (a period that has been referred to as the "Russian mortality crisis"). Because this episode also overlapped with Russia's political and economic transition to capitalism and democracy, the underlying cause of the mortality crisis has been subject to considerable debate. However, Bhattacharya et $\mathrm{al}^{27}$ provide a compelling argument that the mortality crisis was mostly attributable to the coincident termination of the Gorbachev antialcohol campaign rather than the political and economic transition.

For the present example, we reanalyze data from Bhattacharya et $\mathrm{al}^{27}$ in 2 ways. First, we conduct a single-group ITSA with a crossover design to evaluate the effect of introducing and then withdrawing the antialcohol campaign on mortality in Russia between the years 1960 and 2005. Next, we conduct separate multiple-group ITSAs to compare the mortality rates in Russia to those of 3 groups of countries that would be expected to have a change in mortality rates proportionate with their exposure to the antialcohol campaign and ethnic/ religious composition. The first group consists of former Baltic/Western Soviet states that were exposed to the campaign and have a small share of Muslims (Latvia, Lithuania, Estonia, Ukraine, Belarus, and Moldova). The second group consists of former Soviet States that were exposed to the campaign and have a large share of Muslims (Armenia, Azerbaijan, Georgia, Uzbekistan, Kazakhstan, Krygyzstan, and Turkmenistan), and the third group consists of non-Soviet Eastern European countries that were not exposed to the antialcohol campaign at all but did undergo a similar political and economic transition (the Czech Republic, the Slovak Republic, Hungary, and Poland). ${ }^{27}$ Considering the ethnic composition is important in this analysis because Islam prohibits the use of intoxicants, and thus the antialcohol 
campaign would not be expected to significantly reduce mortality in countries with large Muslim concentrations. ${ }^{27}$

In all analyses, the preintervention phase (ie, pre antialcohol campaign) spans from 1960 to 1984; the intervention phase spans from 1985 to 1990; and the postintervention phase spans from 1991 to 2005 (3 distinct phases). As in the previous example, all statistical analyses were conducted using ITSA, ${ }^{9,10}$ and for comparisons between groups, the time series were ipsatively standardized to allow for comparisons between groups on the same scale.

As illustrated in Figure 2, deaths per thousand increased linearly in Russia from 1960 to 1985 . There was a sharp drop in deaths immediately after the introduction of the antialcohol campaign in 1986, and the level appears attenuated until 1991, at which point annual deaths returned to the same level and trend of the time series before the antialcohol campaign. The results from this single-group ITSA indicate that overall mortality rates in Russia decreased during the period of the antialcohol campaign. However, this design does not provide an unambiguous answer as to whether the decrease in mortality rates was causally associated with the campaign, and whether the sharp rise in mortality rates starting in 1991 was causally associated with the withdrawal of the campaign.

Figure $2 \mathrm{~B}$ shows the comparison of annual mortality rates in Russia versus other former Soviet states that were also exposed to the campaign and have low Muslim concentrations. As expected, these states exhibited a trend in overall mortality similar to that of Russia, thereby confirming that the antialcohol campaign was equally effective in other states exposed to intervention with a similar demographic composition.

Figure $2 \mathrm{C}$ shows the comparison of annual mortality rates in Russia versus other non-Soviet Eastern European countries that were not exposed to the antialcohol campaign but did undergo a political and economic transition as well. As illustrated, mortality rates in nonSoviet countries rose over time before the campaign (between 1960 and 1985) then flattened during the period of the campaign, but in contrast to Russia, it declined after 1991. Although this may call into question the causal relationship between campaign and mortality (given that both Russia and non-Soviet Eastern bloc countries experienced a flat trend in mortality during the campaign years), it does suggest that withdrawal of the campaign was more causally associated with the Russian mortality crisis than the political and economic transitions (given that the transition occurred across all these countries contemporaneously).

Figure 2D shows the comparison of annual mortality rates in Russia versus those of former Soviet states with higher concentrations of Muslims. As hypothesized, the trend in mortality rates of the Central Asia/Caucasus countries followed a completely different pattern than that of Russia. In these former Soviet states, mortality rates declined from 1960 to 1990 and then appeared to stabilize over the remaining duration of the study. If the Russian mortality crisis was entirely due to the political and economic transition and not withdrawal of the antialcohol campaign, we would expect to see a comparable increase in mortality in this set of former Soviet states
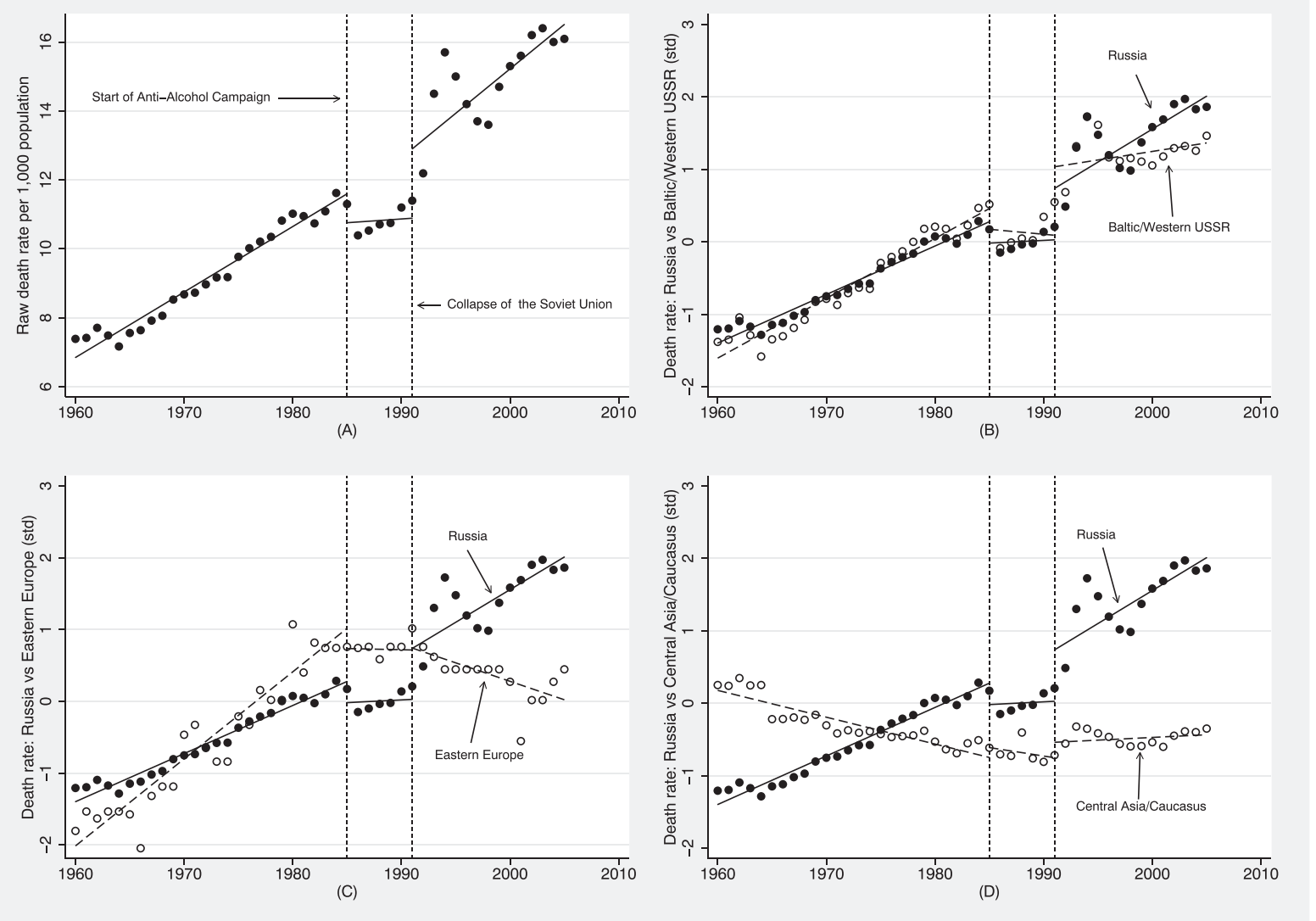

FIGURE 2 Raw death rates per 1000 population in Russia and other former Soviet and non-Soviet states from 1960 to 2005 , using single-group and multiple-group ITSA designs 
that also experienced the transition but were not sensitive to the antialcohol policy.

In summary, this example again highlights the effect of history in complicating the interpretation of ITSA results-even when utilizing a crossover design. Only when contrasting the results against those of a control group (or in this case, against different sets of control groups) was the treatment effect of Gorbachev's antialcohol campaign substantiated, and relatedly, the withdrawal of the campaign was found to be the more likely culprit in explaining the Russian mortality crisis than Russia's transition to capitalism and democracy.

\section{5 | DISCUSSION}

The 2 examples presented in this paper suggest that the single-group ITSA crossover design is just as vulnerable to the effects of history as the basic single-group ITSA design. ${ }^{6}$ In the first example, a seemingly unquestionable treatment effect (and withdrawal effect) across multiple crossovers was reversed when contrasted with a comparable control group. In the second example, a negative change in the time series after withdrawal of the intervention was attributed to an external event, and only when compared with a control group did the withdrawal of the intervention receive correct attribution for that effect. In short, even with the addition of 1 or more crossovers, a single-group ITSA remains susceptible to threats to validity that limit the ability to draw causal inferences about the effects of the intervention.

As demonstrated in the present examples (in addition to those presented in Linden ${ }^{6}$ ), using a control group to serve as the counterfactual is the most robust approach for assessing treatment effects. Only when contrasted with a comparable control group can the effect of the intervention (or withdrawal of the intervention) be isolated from other rival factors. Moreover, other anomalies observed in the time series (eg, changes in instrumentation, selection bias) can alert the investigator to other potential sources of confounding.

When multiple nontreated units are available, investigators can choose from at least 3 different matching methods suitable for time series data. This includes the matching process implemented in the present examples (ie, finding nontreated units that are nonstatistically different from the treated unit on preintervention levels and trend of the outcome variable), ${ }^{9}$ a synthetic controls approach ${ }^{28}$ or propensity score-based weighting ${ }^{10}$ (which can also be extended to situations in which multiple treated units are available) ${ }^{29}$ and for censored data. ${ }^{30,31}$ Investigators should also consider the use of an instrumental variables strategy in cases where some of the right-hand side covariates are endogenous. ${ }^{32-34}$ Most statistical software packages have commands designed to implement these approaches (such as XTIVREG in Stata).

Finally, although this paper has illustrated that the crossover design does not ensure improved validity when implemented in a single-group study, the crossover design can further enhance the robustness of an ITSA study that includes a comparable control group to serve as the counterfactual. In such a study, the groups switch their treatment assignment at a given time point (ie, the treatment group switches to control and the control switches to treatment) and the outcomes change in accordance with the exposure to the intervention. Although clearly difficult to implement in practice, the design, when properly executed, may possibly be considered as good as randomized (see Barlow et $\mathrm{al}^{35}$ and Biglan et $\mathrm{al}^{36}$ for other ITSA design alternatives to improve causal inference over the basic single-group design).

In summary, this paper illustrated that history-the foremost threat to validity in the basic single-group ITSA design-persists even when adding 1 or more treatment crossovers to the study. Absent a comparable control group as a contrast, there is simply no assurance that the effect of external factors have been identified and controlled for, regardless of whether the time series follow the expected pattern after each crossover. Thus, even when using a single-group ITSA crossover design, the results should be considered preliminary-and interpreted with caution-until a more robust study design can be implemented. Given the popularity and widespread use of single-group ITSA designs, it is important for investigators to be cognizant of their limitations and to strive to add a comparable control group to maximize validity and improve causal inference.

\section{ACKNOWLEDGMENTS}

The author thanks Grant Miller for providing the data used in the second example of this paper. He also thanks Julia Adler-Milstein for reviewing the manuscript and for providing many helpful comments.

\section{REFERENCES}

1. Campbell DT, Stanley JC. Experimental and Quasi-Experimental Designs for Research. Chicago, IL: Rand McNally; 1966.

2. Shadish WR, Cook TD, Campbell DT. Experimental and QuasiExperimental Designs for Generalized Causal Inference. Boston: Houghton Mifflin; 2002.

3. Linden A. Estimating the effect of regression to the mean in health management programs. Dis Manag Health Out. 2007;15:7-12.

4. Linden A. Assessing regression to the mean effects in health care initiatives. BMC Med Res Methodol. 2013;13:1-7.

5. Linden A, Adams J, Roberts N. The generalizability of disease management program results: getting from here to there. Manag Care Interface. 2004;17:38-45.

6. Linden A. Challenges to validity in single-group interrupted time series analysis. J Eval Clin Pract. 2017;23:413-418.

7. Rubin DB. Estimating causal effects of treatments in randomized and nonrandomized studies. J Educ Psychol. 1974;66:688-701.

8. Rubin DB. Causal inference using potential outcomes: design, modeling, decisions. J Am Stat Assoc. 2005;100:322-331.

9. Linden A. Conducting interrupted time-series analysis for single- and multiple-group comparisons. Stata J. 2015;15:480-500.

10. Linden A, Adams JL. Applying a propensity-score based weighting model to interrupted time series data: improving causal inference in program evaluation. J Eval Clin Pract. 2011;17:1231-1238.

11. Linden A, Adams JL. Combining the regression-discontinuity design and propensity-score based weighting to improve causal inference in program evaluation. J Eval Clin Pract. 2012;18(2):317-325.

12. Linden A, Yarnold PR. Using machine learning to identify structural breaks in single-group interrupted time series designs. J Eval Clin Pract. 2016;22:851-855.

13. Linden A, Roberts N. Disease management interventions: what's in the black box? Dis Manag. 2004;7(4):275-291.

14. Linden A, Butterworth S, Roberts N. Disease management interventions II: what else is in the black box? Dis Manag. 2006;9(2):73-85. 
15. Box GEP, Tiao GC. Intervention analysis with applications to economic and environmental problems. J Am Stat Assoc. 1975;70:70-79.

16. Glass GV, Willson VL, Gottman JM. Design and Analysis of Time-Series Experiments. Boulder: University of Colorado Press; 1975.

17. McDowall D, McCleary R, Meidinger EE, Hay RA. Interrupted Time Series Analysis. Newbury Park, CA: Sage Publications, Inc; 1980.

18. Crosbie J. (1993) Interrupted time-series analysis with brief singlesubject data. J Consult Clin Psychol. 1993;61:966-974.

19. Gottman JM. Time-series analysis. A Comprehensive Introduction for Social Scientists. New York: Cambridge University Press; 1981.

20. McKnight S, McKean JW, Huitema BE. A double bootstrap method to analyze linear models with autoregressive error terms. Psychol Methods. 2000;5:87-101.

21. Simonton DK. Cross-sectional time-series experiments: some suggested statistical analyses. Psychol Bull. 1977;84:489-502.

22. Velicer WF, McDonald RP. (1991) Cross-sectional time series designs: a general transformation approach. Multivariate Behav Res. 1991;26:247-254.

23. Chaudhary GH, Neil K, Solomon MG, Preusser DF, Cosgrove LA. Evaluation of the Reinstatement of the Helmet Law in Louisiana. National Highway Traffic Safety Administration. 2008. Available at: www. nhtsa.gov/DOT/NHTSA/Traffic\%2OInjury\%20Control/Articles/Associated\%20Files/810956.pdf. Accessed September 7, 2016.

24. U.S. Department of Transportation, National Highway Traffic Safety Administration, National Center for Statistics and Analysis. Fatality Analysis Reporting System (FARS). Available at: ftp://ftp.nhtsa.dot. gov/fars/. Accessed September 7, 2016.

25. U.S. Department of Transportation, Federal Highway Administration. Highway Statistics (Multiple Years). Available at: http://www.fhwa. dot.gov/policyinformation/statistics.cfm. Accessed on September 7, 2016.

26. Yarnold PR, Linden A. Using machine learning to model dose-response relationships via ODA: eliminating response variable baseline variation by ipsative standardization. Optimal Data Analysis. 2016;5:41-52.
27. Bhattacharya J, Gathmann C, Miller G. The Gorbachev anti-alcohol campaign and Russia's mortality crisis. Am Econ J Appl Econ. 2013;5:232-260.

28. Abadie A, Diamond A, Hainmueller J. Synthetic control methods for comparative case studies: estimating the effect of California's tobacco control program. J Am Stat Assoc. 2010;105:493-505.

29. Linden A, Adams JL. Evaluating health management programmes over time. Application of propensity score-based weighting to longitudinal data. J Eval Clin Pract. 2010;16:180-185.

30. Robins JM, Hernán MA, Brumback B. Marginal structural models and causal inference in epidemiology. Epidemiology. 2000;11:550-560.

31. Linden A, Adams J, Roberts N. Evaluating disease management program effectiveness: an introduction to survival analysis. Dis Manag. 2004; 7:180-190.

32. Linden A, Adams J. Evaluating disease management program effectiveness: an introduction to instrumental variables. J Eval Clin Pract. 2006;12(2):148-154.

33. Baltagi BH. Econometric Analysis of Panel Data. 4th ed. New York: Wiley; 2008.

34. Becketti S. Introduction to Time Series Using Stata. College Station, TX: Stata Press; 2013.

35. Barlow DH, Hayes SC, Nelson RO. The Scientist Practitioner: Research and Accountability in Clinical and Educational Settings. New York: Pergamon Press; 1984.

36. Biglan A, Ary D, Wagenaar AC. The value of interrupted time-series experiments for community intervention research. Prev Sci. 2000;1:31-49.

How to cite this article: Linden A. Persistent threats to validity in single-group interrupted time series analysis with a cross over design. J Eval Clin Pract. 2017;23:419-425. https://doi. org/10.1111/jep.12668 